\title{
25 Research Square \\ Pattern and determinants of antibiotic mal-prescribing among family physicians in the primary health care facilities in Iran
}

\section{Gholamali Karimi}

Alborz University of Medical Sciences

Hosein Azizi ( $\square$ aziziepid@gmail.com )

Tabriz University of Medical Sciences https://orcid.org/0000-0002-4163-6158

Kourosh Kabir

Alborz University of Medical Sciences

\section{Babak Farrokhi}

Tehran University of Medical Sciences

Ali Delpisheh

Ilam University of Medical Sciences

Effat Abbaszadeh

Alborz University of Medical Sciences

Elham Davtalab Esmaeili

Tabriz University of Medical Sciences

\section{Research article}

Keywords: Antibiotics prescription, Family physicians, Primary health care, Mal-prescribing, Iran

Posted Date: December 4th, 2019

DOI: https://doi.org/10.21203/rs.2.18183/v1

License: (9) (i) This work is licensed under a Creative Commons Attribution 4.0 International License. Read Full License 


\section{Abstract}

Background Mal-prescribing of antibiotics is a major and ongoing global public health problem both in developing and developed countries. Family physicians are the most important and dominant health services providers in Iran that pattern describing by them poorly understood. This study aimed to assess the pattern and factors affecting antibiotic prescribing by family physicians in primary health care (PHC).

Methods In this descriptive-analytical study, 1068 prescriptions of family physicians in PHC were assessed among Alborz province health facilities. Prescriptions were selected by random sampling. The mal-prescribing was assessed based on 4 criteria including dose per consumption, dose per day, correct duration of therapy and possible interaction with other antibiotics. Logistic regression was used to estimate the odds ratio and $95 \%$ confidence interval for the association between antibiotic prescribing and affecting factors.

Results The average number of antibiotics was reported 1.27 per prescription and almost $56.8 \%$ of them had at least an antibiotic. There was a statistically significant relationship between age, gender, type of insurance booklet, work experience of physicians, seasons of the year and receiving antibiotics $(P<0.05)$. In $59.31 \%$ of antibiotic prescriptions at least one of the scientific criteria was not satisfied.

Conclusion The number of prescribed antibiotics and the mal-prescribing percentage were high among family physicians. Strong political commitment and altering physicians' training curricula especially promoting a preventive approach and developing a registration system for identifying adverse drug reactions, antibiotic use patterns and mal-prescribing are imperative at the grass-root level.

\section{Introduction}

Irrational use and mal-prescribing of drugs are a major and ongoing global public health problem both in developing and developed countries that deserves more attention by health systems and policymakers $(1,2)$. Rational and appropriate usage of drugs were defined, the reasonable and proposed use of drugs at an advisable time so that they have had beneficial effects on patients in terms of the virtue of strength, dose and duration of therapy (3). Rational use of drugs depends on pursuing the process of prescription which includes identification of patients' problem (diagnosis), effective and safe therapy (therapy with drugs or non-drugs options), selecting suitable drugs, dosage, and duration, writing a good prescription, providing enough information to the patient and planning to evaluate treatment responses (4).

Self-medication by patients, prompt and constant spread of antimicrobial-resistant organisms are the major threat to our ability to successfully treat growing many contagious diseases. In the lack of the development of new generations of antibiotic drugs, appropriate use of current antibiotics is required to guarantee the long-term availability of effective therapy for microbial infections $(2,5-7)$.

In Iran, Primary Health Care (PHC) is the first, nearest and comprehensive line of basic and essential health services in the Iranian health system provided by family physicians and many types of health workers in all cities and villages. In Iran, some evidence indicated that the inappropriate prescription of antibiotics and large self-medication is one of the main causes of antibiotics resistance and side reactions $(8,9)$. In Iran, antibiotics are used common drugs, and almost half of the patients during doctor-patient encounters received at least an antibiotic so that it is estimated that more than $8 \%$ of hospital admission in Iran occur due to adverse drug reactions $(5,10,11)$. So, rational and appropriate drug prescribing especially antibiotics will decrease antibiotics resistance at the global level, adverse drug reactions, toxicity risks, healthcare expenditure and household costs, and duration of therapy $(11,12)$.

Among PHC family physicians, pattern and factors associated with mal-prescribing poorly understood and very limited studies have been performed especially after execution of rural insurance and family physician plan, the present study has been done by goal of determination of the prescribing pattern of antibiotics for outpatients in therapeutic and health centers and also determining the effective factors on this pattern. 


\section{Methods \\ Study design}

In this descriptive-analytical study, 1068 prescriptions by 48 family physicians (267 prescriptions in each season) in PHC were selected among total 90115 prescriptions from 8 rural, 8 urban and 3 urban-rural health centers from September 2012 to September 2013 in Alborz province of Iran.

Prescriptions were selected by random sampling and considering proportional to the size of Iranian insurance booklet types. In the sampling process, the unreadable prescription was excluded and the next prescription was replaced. The sample size was estimated in 1068 by considering $a=0.05$ and $d=0.03$ and $p=0.5$.

\section{Data collection}

Incorrect or mal-prescribing of antibiotics was assessed based on 4 scientific items including a) dose per consumption, b) dose per day, c) duration of therapy and d) possible interaction with other antibiotics or drugs. The incorrect prescription was defined if at least one of the items above is not satisfied. The prescribed assessment was performed by a high expert (more than 10 years' experience) pharmacy based on Martindale: The Complete Drug Reference.

A checklist was used for data collection. Variables and information such as age, gender of physicians and patients, number and total price of drug items and prescribed antibiotic name and type, form and usage of drug, consumption way based on amount of use for each time, duration of treatment, times of use in each day, possible interaction with antibiotics or drugs, rate of combination therapy, type and kind of physicians' graduation and also the statues of occupation and year of services from prescriptions and personnel files were collected.

\section{Data analysis}

SPSS software (version 18.0, Chicago, IL, USA) was used for data analysis. For checking data normality, the KolmogorovSmirnov test was used. Chi-square test was used for binary variables and independent t-test was used for normal quantities variables. Logistic regression was used to estimate the odds ratio and $95 \%$ confidence interval for the association between antibiotic prescribing and affecting factors. P-value $<0.05$ was considered significant in all of the tests.

\section{Results}

Table 1 indicated that some socioeconomic status and factors affecting the relationship with odds of antibiotics prescribing by family physicians in primary health care, Alborz province, Iran. The results showed that a total of 1068 prescriptions which were studied, 607 prescription (56.8\%) had at least one item of antibiotic and 461 prescription (43.2\%) were without any antibiotic and also 3704 items of prescribed drugs in this prescription, 772 items (20.8\%) are specified by antibiotics. It was to be found a significant difference between antibiotic prescribing and different seasons of the year. A total of 267 prescriptions in the summer season, 112 (41.9) of them had an antibiotic while, as the same number of this prescription in winter, 177 prescriptions $(66.3 \%)$, patients had received antibiotics $(p<0.05)$. Among all assessed prescriptions, $640(59.9 \%)$ of them was related to female genders, while the proportion of received antibiotic was more in males than females (63.3\% versus $52.5 \%$ ). Moreover, according to the insurance booklet among 1068 assessed prescriptions, more than $50 \%$ of them prescribed with a social security booklet (table1). This study showed that there is a relation between physicians' experience and the times of antibiotic prescription in such a way that by an increase in experience and year of service, antibiotic prescribing was decreased $(p=0.008)$.

The average age of study outpatients was $32.4 \pm 21.4$ years. Patients who had received antibiotics $(26.07 \pm 19.3)$ with those who didn't receive antibiotics $(40.7 \pm 21.2)$ there was a statistical significant difference (Fig1). Also, the mean age of outpatients 
receiving prescription was reported lowest and highest in winter and summer, respectively (Fig2).

The average drug items and price per prescription were 3.47 \pm 1.3 and $9034 \mathrm{RIs}$, respectively and in prescription consisting antibiotic was $3.67 \pm 1.22$, and Rls. 35065 , respectively. It was to be found a significant association between both groups of prescription with and without antibiotic (Table 2).

Table 3 demonstrated that the distribution of prescribed antibiotics based on name, the form of drug and consumption way by family physicians, Amoxicillin capsule 500 mli.g with $10.2 \%$, pill with $24.7 \%$ and oral method of consumption with $70.59 \%$ were the most abundant.

Table 4 indicated that the percent and distribution of incorrect prescribing among all 1068 assessed prescription by family physicians. According to our results, incorrect prescribing in a dose per day criteria had the most frequency in total prescriptions both with and without antibiotics with $67.72 \%$.

\section{Discussion}

This study investigated the pattern of antibiotic prescription and it's affecting factors and also the mal-prescribing situation in the Iranian PHC system by family physicians. Family physicians are the most important with high numbers and key health services providers in Iran's PHC. The modification of the antibiotic prescribing pattern by this group of providers can play a major role in reducing the burden of microbial resistance and health expenditures in Iran and global health systems. On the other hand, previous studies had examined most the prescribing of antibiotics in general practitioners, specialists or dentists $(13,14)$. This study is one of the few studies focused on family physicians, at least in Iran.

The percent of prescriptions with an antibiotic in this study (56.8\%) is similar to the result of Indian study with 55\% (15) but this ratio in a study in Sabzevar, Iran was reported $45 \%$ (16).

It is a high percentage of antibiotic prescribing in our study and the reason can be the high rate of irrational prescribing by family physicians, beliefs and different social and cultural factors among different level of people, high rate of environmental polluting materials in accordance to the industrial zone and specially air pollution because of neighboring to the metropolitan and Iranian capital such as Karaj and Tehran and suspecting infectious disease such as sinusitis and pharyngitis. Moreover, it may occur due to easy access to medication and drugs and the low price of drugs in comparison to other countries.

However, the rate of antibiotic prescription in our study is less than a study conducted from Tehran metropolitan city and capital of Iran (62.39\%) (17), which supports the effect of accessibility factor and the role of environmental polluting materials is causing more infections.

The average rate of prescribed drug items in this study was 3.47 per prescription, this finding is almost in agreement with Iranian protocol of family physicians prescriptions that is 3.5 items (18), however, this amount is more than from 12 developing countries which is between 2.2 to 3.8 and developed countries about 1.3 to 2.2 , is recorded $(19,20)$, and it is mention that an undesirable statues of drug prescribing in this study.

Another related factor to the antibiotic prescribing in the present study was the impact of the seasons so that the number of antibiotic prescriptions increased as the cold season increased. This finding is in agreement with national studies $(11,21)$.

One of the most important criteria for evaluating the correct and rational prescribing of antibiotics is compliance with international valid guidelines and up-to-date medical science. Our findings showed that a large volume of antibiotics prescribed in our study area did not follow the correct scientific method. This defect may be a major determinant of the development of antibiotic resistance and maybe a threat to human life.

In this study, incorrect and unscientific antibiotic prescribing in prescriptions was assessed based on four criteria including antibiotic dosage per consumption, doses per day, duration of therapy and interaction effect with other antibiotics or drugs. Our findings showed that family physicians prescribed antibiotics in most prescriptions were unscientific or incorrect. This 
finding has been observed in other national studies and other countries (22-26). But this issue in our study was reported slightly high. Therefore, reducing the number of antibiotics in each prescription and inappropriate and unscientific prescribing of antibiotics is a major concern for drug resistance and it is a challenge for the country's health system that deserves tangible attention in improving and modifying it.

In the present study injecting form of antibiotics was prescribed more than other forms with $22.27 \%$ although this amount was reported $49 \%$ in a study in Urmia city (27) that is higher than our study. But the injection form was reported less than our study in the Bhopal zone of India with $13.8 \%$ (28). Therefore, a high percentage of injectable antibiotics are prescribed by family physicians shows an irregular form of injecting antibiotics by them. It may have resulted from cultural-social factors and believing the high effect of injecting form of drugs by patients which besides being expensive in regard to oral form, sometimes is dangerous for patients (29), another reason maybe is the existence of injecting section in most of therapeutic and health centers and following the desire of patients to receive whole services from the place of their refer $(11,17)$. Amoxicillin and Penicillin were the most commonly prescribed drug in our study and a study of Dong and et al. in China (30).

The price of antibiotics in proportion to total price of drug prescriptions were $33.7 \%$ and to the prescriptions included antibiotics $56.4 \%$ which in comparison to the results of a study from Urmia city with $35 \%$ (27) and the finding from other countries such as France with $34.7 \%$ and USA with $33 \%$ was a high number $(31,32)$, so, this is a high rate of antibiotic prescribing and imposing more economic load on families and medicine system of country, based on studies, up to $\$ 3500$ can be saved by limiting the Antibiotic prescribing (33), Anyway the average price of prescriptions in this study which was Rls. 59034 in comparison to the U.S, that was $\$ 75$ in the year 2006, was a low number that shows the less price of the drug and unreal cost of it in-country (34).

\section{Conclusion}

Our findings indicated that the number of prescribed antibiotics and the incorrect prescription proportion are high among family physicians and need intervention. This study can provide useful information about the antibiotic prescribing pattern and mal-prescribing of drugs in Iranian PHC by family physicians. Our study results to assist program managers and policymakers to develop effective strategies to improve irrational antibiotic prescribing patterns and health expenditure in Iranian PHC and also worldwide health systems.

Developing a registration system for identifying adverse drug reactions, antibiotic use patterns, and inappropriate prescriptions, revising in treatment protocols and family physicians purchase services, development of targeted and effective training programs at different levels are suggested.

\section{Abbreviations}

PHC: Primary Health Care

\section{Declarations}

\section{Acknowledgments}

Hereby announce our thanks and appreciate from the cooperation of respectful Deputy of technology and researches and student's researches committee of Medical Sciences University of llam for financial support of project and also Mr. Ali Khosravi, respectfully expert of insuring of Health Center of Savojbolagh city and other experts related that cooperated in collection and exploitation of data.

\section{Authors' contributions}


HA, GK: Developed the original idea, protocol development and interpretation, data analysis, and data collection and drafted all sections of the manuscript. Data collection, Data extraction, contributed to the development of the protocol: $A D, K K, B F, E A$ and EDE. All the authors approved the final manuscript.

\section{Funding}

This study was approved and funded by llam University of Medical Sciences and a grant to number 918087/96.

\section{Ethics approval and consent to participate}

This study was approved by the Student Research Committee and the ethic committee of llam University of Medical Sciences to number IR.MEDILAM.REC.1391/8087 and conducted from drug prescriptions by family physicians. Human samples have not been used for the study.

\section{Consent for publication}

The authors and funding organizations are Consent for publication.

\section{Competing interests}

The authors have no conflicts of interest to declare for this study.

\section{References}

1. Ahmed1, 2 S, Khan S, Alia M, Noushad S. PSYCHOLOGICAL IMPACT EVALUATION OF EARLY MARRIAGES. 2013.

2. Ogunleye OO, Fadare JO, Yinka-Ogunleye AF, Anand Paramadhas BD, Godman B. Determinants of antibiotic prescribing among doctors in a Nigerian urban tertiary hospital. Hospital Practice. 2019;47(1):53-8.

3. Promoting rational use of medicines : core components (WHO policy perspectives on medicines). Geneva: World Health Organization; 2002. Available from : http://www.apps.who.int/medicinedocs/pdf/h3011e/h3011e.pdf. [Last cited 2011 Jun 17]. Back to cited text no. 5

4. De Vries TP, Henning RH, Hogerzeil HV, Fresle DA. Treating your patients. Guide to Good Prescribing: A Practical Manual. Geneva: World Health Organization; 1994 (WHO/DAP/94.11).p.33-66. Available from :

http://www.archives.who.int/prduc2004/rducd/INRUD_2000_CDROM/Manuals/Guide\%20to\%20Good\%20Prescribing.doc. [Last cited 2011 June17].

5. Safaeian L, Mahdanian A-R, Hashemi-Fesharaki M, Salami S, Kebriaee-Zadeh J, Sadeghian G-H. General physicians and prescribing pattern in Isfahan, Iran. Oman medical journal. 2011;26(3):205.

6. Kritsotakis El, Gikas A. Surveillance of antibiotic use in hospitals: methods, trends and targets. Clinical Microbiology and Infection. 2006;12(8):701-4.

7. Vlahovic-Palcevski V, Morovic M, Palcevski G. Antibiotic utilization at the university hospital after introducing an antibiotic policy. European journal of clinical pharmacology. 2000;56(1):97-101.

8. AbdollahiasI A, Kebriaeezadeh A, Nikfar S, Farshchi A, Ghiasi G, Abdollahi M. Patterns of antibiotic consumption in Iran during 2000-2009. International journal of antimicrobial agents. 2011;37(5):489.

9. Hashemi S, Nasrollah A, Rajabi M. Irrational antibiotic prescribing: a local issue or global concern? EXCLI journal. 2013;12:384.

10. Rashidi K, Senobar Tahaee SN. Assessment of drug interactions in medical insurance prescriptions in Kurdistan province in 2000. Scientific Journal of Kurdistan University of Medical Sciences. 2005;10(3):78-84. (Article in persian). 
11. Safaeian L, Mahdanian A-R, Salami S, Pakmehr F, Mansourian M. Seasonality and physician-related factors associated with antibiotic prescribing: A cross-sectional study in Isfahan, Iran. International journal of preventive medicine. 2015;6.

12. Gholamreza-Sepehri, Meimandi M-S. The quality of prescribing in general practice in Kerman, Iran. International journal of health care quality assurance. 2005;18(5):353-60.

13. Dar-Odeh NS, Abu-Hammad OA, Al-Omiri MK, Khraisat AS, Shehabi AA. Antibiotic prescribing practices by dentists: a review. Therapeutics and clinical risk management. 2010;6:301.

14. Davey P, Brown E, Charani E, Fenelon L, Gould IM, Holmes A, et al. Interventions to improve antibiotic prescribing practices for hospital inpatients. Cochrane Database of Systematic Reviews. 2013(4).

15. Gopalakrishnan S, Ganeshkumar P, Katta A. Assessment of prescribing practices among urban and rural general practitioners in Tamil Nadu. 2013;45(3):252-7.

16. Dolatabadi M, Jalili Rasti H. PATTERNS OF PHYSICIANS' DRUG PREH1ION IN SABZEVAR IRAN (2008). 2. 2009;16(3):1616. (Article in Persian).

17. Mosleh A, Darbooy SH, Khoshnevis Ansari SH, Mohammadi M. Drug prescription based on WHO indicators: Tehran university of medical sciences facilities with pharmacy. Tehran University Medical Journal. 2008;65(14):12-5. (Article in persian).

18. Ahadian M. Self medication and drug abuse. Journal of drug nedaye mahya. 2007;1(3):14-35. (Article in Persian).

19. Ofori-Adjei D. Report on Tanzania field test. INRUD(International Network for Rational Use of Drugs), News. 1992:9-10.

20. Gilson L, Jaffar S, Mwankusye S, Teuscher T. Assessing prescribing practice: A tanzanian example. The International Journal of Health Planning and Management. 1993;8(1):37-58.

21. Sobhani A, Shojaei H, Pasandideh M, Pourreza B. Evaluation of drug prescription in Guilan. The Journal of Qazvin University of Medical Sciences. 1999;2(4):11-6. (Article in persian).

22. Alavi Moghaddam M, Yadegarinia D, Zamiri SA. Pattern of Empiric Antibiotic Prescription in Patients Referred to an Emergency Department of a Medical University Affiliated Hospital in Tehran. Pejouhandeh. 2009;14(1):31-6. (Article in persian).

23. Moghadamnia A, Mirbolooki M, Aghili M. General practitioner prescribing patterns in Babol city, Islamic Republic of Iran. 2002.

24. Bharathiraja R, Sridharan S, Chelliah LR, Suresh S, Senguttuvan M. Factors affecting antibiotic prescribing pattern in pediatric practice. Indian journal of pediatrics. 2005;72(10):877-9.

25. Dumpis U, Dimiṇa E, Akermanis M, Tirāns E, Veide S. Assessment of antibiotic prescribing in Latvian general practitioners. BMC Fam Pract. 2013;14(1):1-5.

26. Fahimzad A, Eydian Z, Karimi A, Shiva F, Sayyahfar S, Kahbazi M, et al. Surveillance of antibiotic consumption point prevalence survey 2014: Antimicrobial prescribing in pediatrics wards of 16 Iranian hospitals. Archives of Iranian medicine. 2016;19(3):204-9.

27. Aghayar Makoei A, Gharehaghaji R, Saberi A. General practiyioners pattern of Antibiotic prescription in ambulatory patients in Urimia (1998). URMIAMJ. dec2003;13(4):9-15. (Article in persian).

28. De Costa A, Bhartiya S, Eltayb A, Nandeswar S, Diwan VK. Patterns of drug use in the public sector primary health centers of Bhopal district. Pharm World Sci. 2008 Oct;30(5):584-9.

29. McLaws M-L, Ghahramani S, Palenik CJ, Keshtkar V, Askarian M. Assessment of injection practice in primary health care facilities of Shiraz, Iran. American journal of infection control. 2014;42(3):300-4.

30. Dong L, Yan H, Wang D. Antibiotic prescribing patterns in village health clinics across 10 provinces of Western China. Journal of Antimicrobial chemotherapy. 2008;62(2):410-5.

31. Belghith Z, Khayeche F, Soltani MS, Ben Salem K, Bchir A. Antibiotic therapy in general medicine in Monastir, Tunisia]. East Mediterr Health J. 1999 Mar;5(2):328-32.

32. Mainous IA, Hueston WJ, Love MM. Antibiotics for colds in children: Who are the high prescribers? Archives of Pediatrics \& Adolescent Medicine. 1998;152(4):349-52.

Page $7 / 10$ 
33. Mandell G, Bennett J, Dolin R. Principles and practice of Infectious Disease. 6th ed: Philadelphia: Elsevier Churchill Livingstone; 2005. 612-17 p.

34. Katzung BG, Masters SB, Trevor AJ. Basic and Clinical Pharmacology. 11th ed: McGraw-Hill Education; 2009. 1136 p.

\section{Tables}

Table1. Some of socio-economic status and factors affecting antibiotic prescription by family physicians in primary health care, Alborz province, Iran

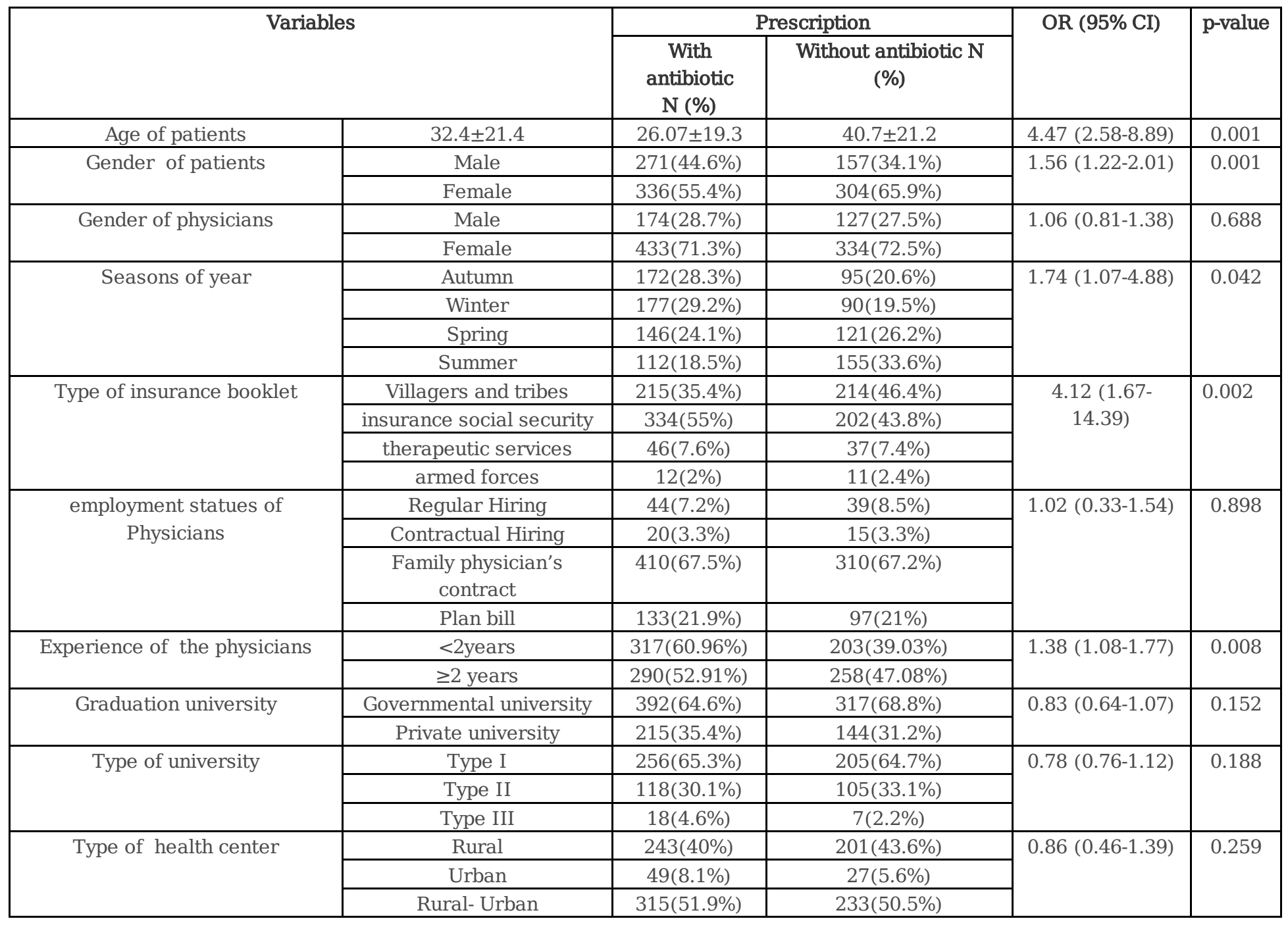

Table 2. The average and difference mean of number and price of total drug items and antibiotic items among prescriptions with and without antibiotic 


\begin{tabular}{|c|c|c|c|c|c|c|c|}
\hline \multirow[t]{2}{*}{ Variables } & \multicolumn{2}{|c|}{ Prescription } & \multirow[t]{2}{*}{ Mean \pm SD } & \multirow[t]{2}{*}{$\begin{array}{c}\mathrm{p}- \\
\text { value }\end{array}$} & \multirow[t]{2}{*}{$\begin{array}{c}\text { Mean } \\
\text { difference }\end{array}$} & \multicolumn{2}{|c|}{$\begin{array}{c}\text { 95\% Confidence } \\
\text { Interval }\end{array}$} \\
\hline & $\begin{array}{c}\text { with } \\
\text { antibiotic }\end{array}$ & $\begin{array}{l}\text { without } \\
\text { antibiotic }\end{array}$ & & & & $\begin{array}{l}\text { Lower } \\
\text { Bound }\end{array}$ & $\begin{array}{l}\text { Upper } \\
\text { Bound }\end{array}$ \\
\hline Total of drug items & 2229 & 1475 & $3.47 \pm 1.3$ & 0.000 & 0.47 & 0.31 & 0.62 \\
\hline Total of antibiotic items & 772 & 0 & $1.27 \pm 76$ & 0.000 & 1.27 & 1.23 & 1.31 \\
\hline Price of all per prescribing & $37,719,350$ & $25,329,210$ & $59034.23 \pm 9161.65$ & 0.018 & 7196.55 & 1260 & 13132 \\
\hline $\begin{array}{l}\text { Price of antibiotics per } \\
\text { prescribing }\end{array}$ & $21,284,955$ & 0 & $35065.19 \pm 4172.86$ & 0.000 & 35065.8 & 32924 & 37207 \\
\hline
\end{tabular}

Table3. Frequency of prescribed antibiotics based on name, the form of drug and consumption method by family physicians

\begin{tabular}{|c|c|c|c|c|c|c|}
\hline Rank & Name & Percentage & Form of Drug & Percentage & Method & Percentage \\
\hline $\mathrm{I}$ & Amoxicillin 500 mil.g (cap) & $10.23 \%$ & Tablet & $24.7 \%$ & Oral & $70.59 \%$ \\
\hline II & Penicillin 1,200,000 u.v (vial) & $9.06 \%$ & Capsule & $23.05 \%$ & Injection & $22.27 \%$ \\
\hline III & Azithromycin 250 mil.g (cap) & $8.03 \%$ & Suspension & $22.7 \%$ & Local & $7.12 \%$ \\
\hline IV & Penicillin 6.3.3 u.v (vial) & $6.9 \%$ & Vail & $21.3 \%$ & & \\
\hline V & Cefixime 100 (susp) & $5.8 \%$ & Ointment & $4.2 \%$ & & \\
\hline VI & Cefixime 400 mil.g (tab) & $5.6 \%$ & Drop & $2.7 \%$ & & \\
\hline VII & Co-amoxyclave 312 (susp) & $5.5 \%$ & Ampoule & $0.9 \%$ & & \\
\hline VIII & Other antibiotics & $48.88 \%$ & & & & \\
\hline
\end{tabular}

Table4. Distribution of incorrect types of prescribed antibiotics by family physicians in primary health care

\begin{tabular}{cccc}
\hline Type of incorrect prescribing & With Antibiotic & Without Antibiotic & Total incorrect \\
\hline Dose per consumption & $10.23 \%$ & $12.52 \%$ & $21.9 \%$ \\
Doses per day & $34.06 \%$ & $38.71 \%$ & $67.72 \%$ \\
\hline Duration of treatment & $15.02 \%$ & $17.13 \%$ & $29.97 \%$ \\
\hline Interaction with other antibiotics & - & $23.22 \%$ & $40.63 \%$ \\
\hline Total & $59.31 \%$ & $91.58 \%$ & - \\
\hline
\end{tabular}

\section{Figures}




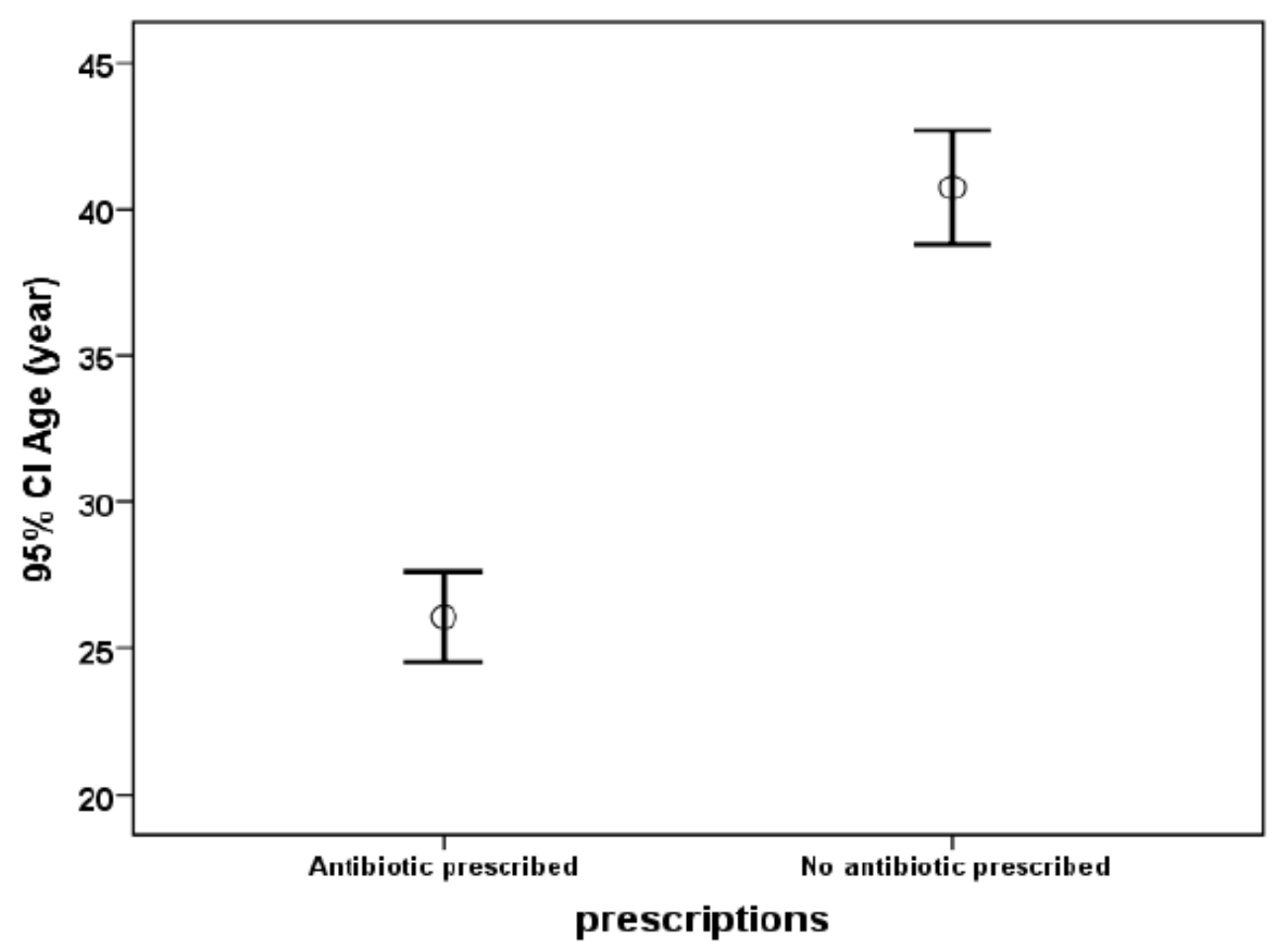

Figure 1

Comparing the difference of average of patients' age in prescription with antibiotic without antibiotic

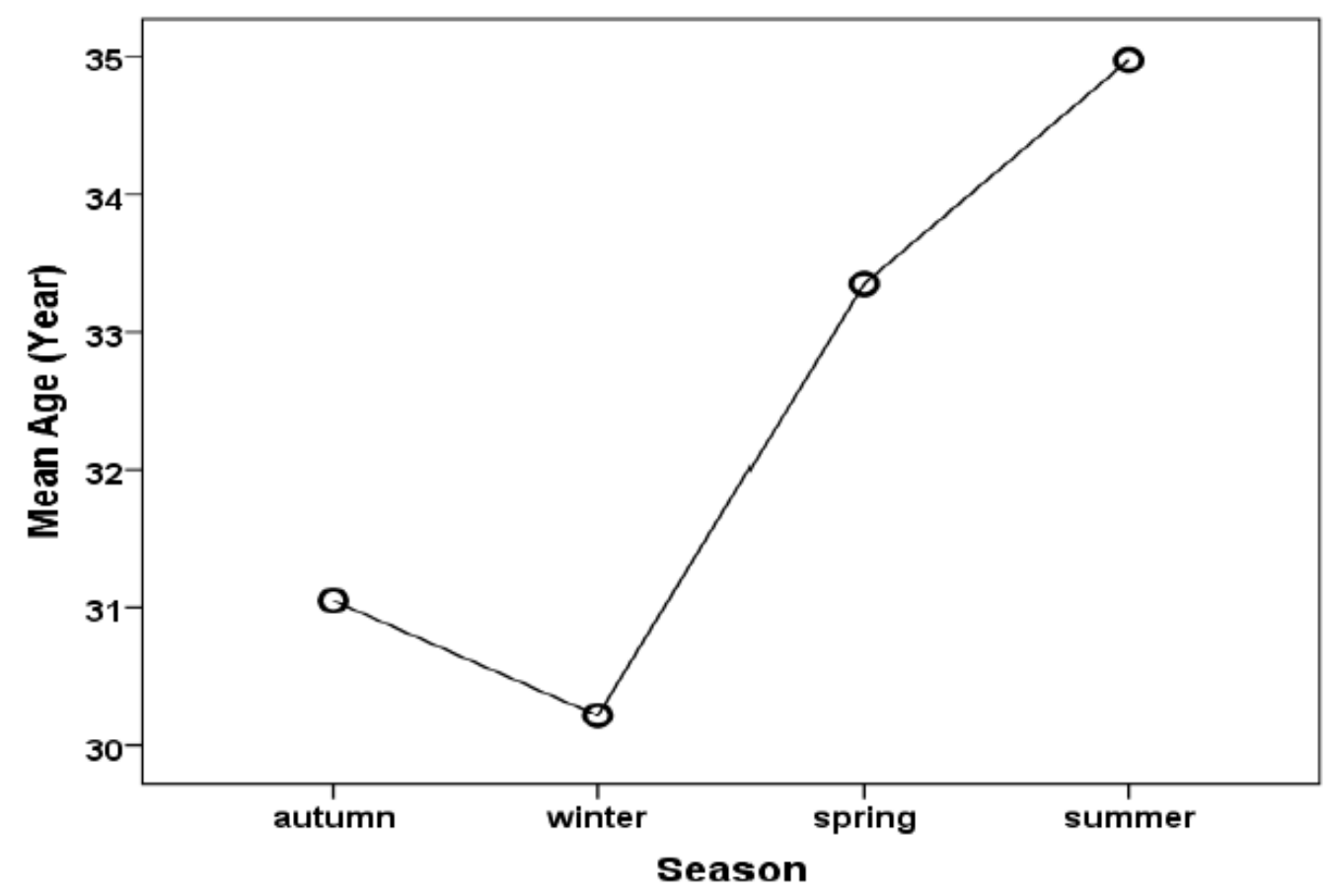

Figure 2

Comparing the difference in the average of patients' age based on seasons 